\title{
Therapeutic outcome of patients suffering from malignant melanomas of the conjunctiva
}

Eye Hospital, Karl Marx University, Leipzig, German Democratic Republic

P K Lommatzsch R E Lommatzsch P Fuhrmann

Institute of Social Hygiene, Humboldt University Berlin (Charité), Otto(Charite), Otto1040 Berlin, German Democratic Republic I Kirsch

Correspondence to: P K Lommatzsch, Klinik fü Augenkrankheiten, KarlMarx-Universität Leipzig, Liebigstrasse 14, 7010 Leipzig, German Democratic Republic.

Accepted for publication 17 May 1990

\author{
P K Lommatzsch, R E Lommatzsch, I Kirsch, P Fuhrmann
}

\begin{abstract}
Eighty-one cases of conjunctival melanoma treated between 1960 and 1988 were studied to determine factors that might affect outcome in patients with such lesions. The therapeutic procedures performed were local excision (16), local excision followed by brachytherapy with Sr-90/Y-90 (32), local excision followed by cryotherapy with liquid nitrogen (16), brachytherapy with Sr-90/Y-90 (12), local excision followed by external beam irradiation (3), and local excision followed by brachytherapy and cryotherapy (2). The median follow-up period was 5.5 years (longest 26, shortest 1 year). Sixty two patients $(\mathbf{7 6} \cdot 5 \%)$ showed a complete regression of the melanoma, $19(23.5 \%)$ developed recurrences, and $15(18.5 \%)$ died from metastases. The melanomas had developed with almost equal frequency from a pre-existing naevus $(25.9 \%)$, from primary acquired melanosis (25.9\%), and 'de novo' (30.9\%). Small tumours had a higher chance of regressing $(80 \cdot 6 \%)$ than larger ones $(68 \cdot 6 \%)$. The cumulative survival rate was $76 \%$ after five years and $60 \%$ after 10 years from any causes of death and $87.6 \%$ after five years and $76.3 \%$ after 10 years from deaths caused by metastases. Most deaths from metastases occurred within 5 years. At $88.5 \%$, the cumulative survival rate of patients with small tumours (less than one quadrant of the bulbar conjunctiva and less than $\mathbf{2} \mathbf{m m}$ thickness) was significantly higher than that of patients with larger tumours (more than one quadrant of the bulbar conjunctiva and/or more than $2 \mathrm{~mm}$ thickness) with $65 \%$ after eight years. Local excision followed by $\beta$ ray irradiation (Sr-90/Y-90) or cryotherapy can be recommended as the treatment of choice. Nevertheless the behaviour of conjunctival melanomas remains unpredictable in individual cases.
\end{abstract}

Primary malignant melanomas of the conjunctiva are rare tumours, constituting only $2 \%$ of eye melanomas. This is why so 'much of what has been written about pigmented lesions of the conjunctiva is either anecdotal, speculative, or controversial'.' The controversial nature of this subject is reflected in different classifications of the lesion, in different modes of treatment, and in the uncertainty of many ophthalmologists in judging the clinical behaviour of any melanotic change of the conjunctiva.

The purpose of this study is to present clinical results obtained from 81 patients suffering from conjunctival melanoma in an attempt to provide a better understanding of the behaviour of these tumours and to raise unanswered questions on this topic.
Material and methods

Eighty one patients suffering from malignant melanoma of the conjunctiva were treated and followed up during 1960-88. All were examined by the authors. Included are patients of the University Eye Hospital (Charité) in Berlin (1960-76), from the Municipal Eye Hospital, Berlin-Buch (1976-81), and the University Eye Hospital, Leipzig (1981-8). In the early 1960s we decided to avoid any mechanical irritation of a suspected conjunctival melanoma. Thus for fear of provoking metastases five patients were treated with $\beta$ irradiation without previous biopsy. Later we changed our mind and a biopsy was taken from all tumours before any radio- and cryotherapy.

The following therapeutic procedures were performed: local excision (16), local excision followed by brachytherapy with $\mathrm{Sr}-90 / \mathrm{Y}-90$ (32), local excision followed by cryotherapy with liquid nitrogen (16), brachytherapy with $\mathrm{Sr}-90$ / Y-90 (12), local excision followed by radiotherapy with high energy electrons (3), and local excision followed by brachytherapy and cryotherapy (2). Altogether, exenteration was performed in six, enucleation in one, and lamellar keratoplasty in four patients.

We used $\beta$ ray applicators of several shapes and sizes to cover the tumour completely. ${ }^{2-5}$ The daily dose applied at the tumour surface was 10 Gy until a total dose of $150-200$ Gy was delivered, depending on the thickness of the neoplasm. The radioactive source has to be brought into direct contact with the tumour after local anaesthesia. The irradiation time for the application of $10 \mathrm{~Gy}$ ranged from $40 \mathrm{~s}$ to $10 \mathrm{~min}$. Cryotherapy was carried out at deep temperatures with liquid nitrogen. Contact freezing with applicators of several shapes (OKG 1) was performed in more extended tumours. A liquid nitrogen spray (OKG 3) was used to perform a triple freeze-thaw cycle. Each freezing time was $10 \mathrm{~s}$ to $1 \mathrm{~min}$ depending on the size of the tumour. Cryotherapy is recommended after surgical excision for treating possible residual invasive cells at the surgical margin. ${ }^{6}$

To describe the extent of the neoplasm the recommendations of the TNM classification were used only in part. ${ }^{7}$ The histological specimens were examined by three pathologists (Professor G Goder of Berlin, Prosektor G Bauke of Berlin, and Dozent U Fuchs of Leipzig). Unfortunately some specimens of the 1960 s were lost before reappraisal.

After treatment all the patients were followed up once a month. Later on, after stabilisation of the treated eye, we saw our patients at least once a year. In cases of death we questioned the family doctors or the local oncology service about the 
cause of death. The cumulative death rate was calculated by the life table method. ${ }^{89}$

Patients who died from metastases proved by necropsy, biopsy, or clinical examination were counted as tumour related deaths. All patients whose tumour shrank completely and in whom no recurrence was observed for at least one year were classified as locally cured cases. All cases without tumour regression or with new tumour formation and new tumour growth after local therapy were described as recurrences. In case of both local cure and of local recurrence metastases occurred.

Large tumours (more than one quadrant of the bulbar conjunctiva and/or more than $2 \mathrm{~mm}$ thick) were compared with small tumours (less than one quadrant of the bulbar conjunctiva and less than $2 \mathrm{~mm}$ thick) to verify the influence of tumour size on the cure rate, recurrence rate, and survival rate. The significance was examined by the $\chi^{2}$ test and Mantel-Haenszel test.

\section{Results}

FREQUENCY AND INCIDENCE

The National Cancer Registry of the GDR (approximately 16.7 million) has 196 malignant melanomas of the conjunctiva on file ( 77 male and 119 female patients), registered between 1960 and 1985. No increase of frequency was noted during this time. Accordingly the incidence of malignant conjunctival melanomas can be assumed to be 0.08 per 100000 population per year.

\section{FOLLOW-UP PERIOD}

The median follow-up period was 5.5 years (minimum one year, maximum 26 years). Forty four patients $(54 \cdot 3 \%)$ could be followed up for more than five years.

\section{AGE AND SEX}

The average age of all 81 patients was 55 years at the time of primary examination (minimum 21, maximum 93 years). The average age of the female patients was 60 years (minimum 31, maximum 93 years) and higher than that of the male patients with 51 years (minimum 21, maximum 78 years). There was a clear preponderance of 52 female patients $(64 \cdot 2 \%)$.

PRIMARY LOCALISATION, ORIGIN, AND TUMOUR SIZE

The primary tumour was located exclusively in the bulbar conjunctiva in 59 cases $(73 \%)$. In addition the cornea was involved in four cases $(5 \%)$, the tarsal conjunctiva in four $(5 \%)$, the fornix in six (7\%), and cornea and fornix in one case. In two patients $(3 \%)$ the primary tumour was confined to the conjunctiva tarsi, and five patients $(6 \%)$ had developed a primary malignant melanoma of the lacrimal caruncle.

The extent of the conjunctival melanomas before treatment was as follows:

Tumour involved maximum one quadrant of the bulbar conjunctiva ............................................ 38 (46.9\%) $(19 \cdot 8 \%)$
Tumour involved fornix, palpebral conjunctiva or caruncle ..... 18 $(22 \cdot 2 \%)$

Tumour extension into eyelid, cornea or orbit ............ 8 $(9.9 \%)$ The extent of invasion could not be assessed .............. $1(1 \cdot 2 \%)$

Taking into consideration the patients' history and the clinical appearance of the lesions; we found the conjunctival melanomas had developed in 38 patients (46.9\%) 'de novo', in $23(28.4 \%)$ from a pre-existing naevus, and in $20(24 \cdot 7 \%)$ from primary acquired melanosis (PAM).

\section{POSTSURGICAL HISTOPATHOLOGICAL EXAMINATION}

The histopathological results of all tumours primarily excised and of all biopsies taken before radio- or cryotherapy were as follows:

Malignant melanoma confined to the conjunctiva and $2 \mathrm{~mm}$ or less in thickness ........................................... $36(44.5 \%)$ Malignant melanoma confined to the conjunctiva but more than $2 \mathrm{~mm}$ in thickness ................................. 20(24.7\%) Malignant melanoma of the conjunctiva with extension in eyelid, cornea, or orbit ...................................... 15 (18.5\%) No malignant melanoma but primary acquired melanosis ........ 1 $(1 \cdot 2 \%)$

No histopathological examination was available ......... 9(11·1\%)

Malignant transformation of a pre-existing naevus or primary acquired melanosis was suspected in 21 cases $(25.9 \%)$ each, while 'de novo' development seemed most likely in 25 cases $(30.9 \%)$. In 13 cases $(16 \cdot 1 \%)$ no information on the origin of the melanoma was available. These numbers differ only insignificantly from those based on clinical appearance.

\section{THERAPEUTIC RESULTS}

In 52 patients $(64 \cdot 2 \%)$ the tumour shrank completely after radiotherapy or did not recur after excision. Pigmentation was no longer traceable.

In another 10 eyes (12.3\%) the tumour disappeared completely, but within the flat scar some pigmentation could be observed. In the remaining 19 cases $(23.5 \%)$ the tumour either recurred after therapy or further growth could not be prevented.

1. Tumour regression. Altogether the conjunctival melanoma in 62 patients $(76.5 \%)$ showed complete shrinkage or, after excision, no recurrence or remnants of the former tumour (Fig 1).

2. Recurrences. In 19 patients the conjunctival melanoma first receded but later recurred locally or tumour new growth remained uninfluenced. After repeated local therapy a complete regression of the recurrent tumour was finally obtained in nine cases. In 10 cases the tumour continued to grow, and in six patients exenteration was eventually necessary. Nine patients in this group died from metastases.

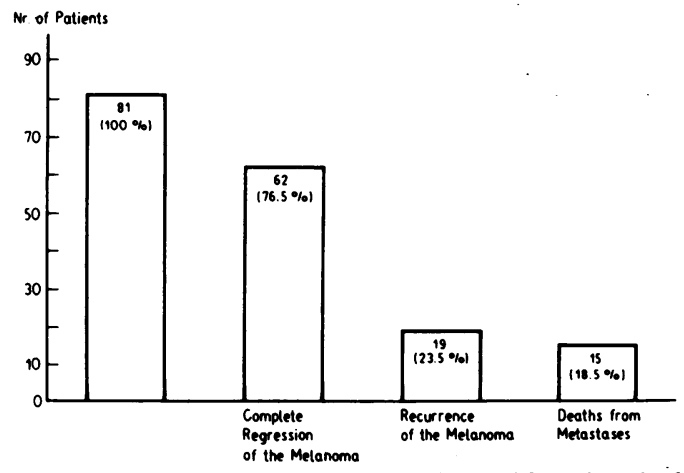

Figure 1 Therapeutic result of 81 patients with conjunctival melanoma (median follow-up 5.5 years). 


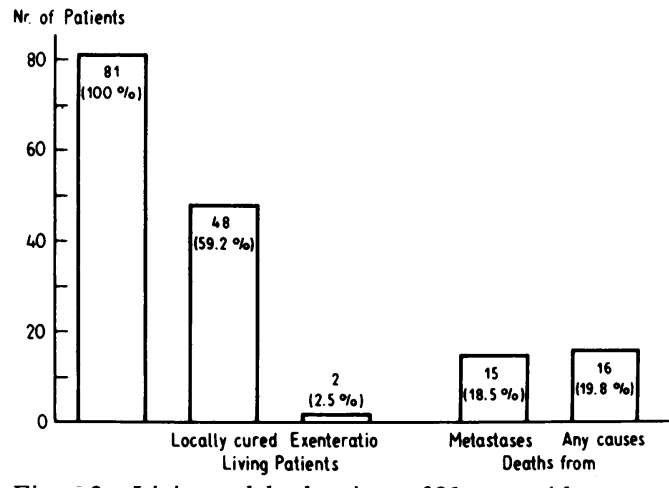

Figure 2 Living and dead patients of 81 cases with conjunctival melanoma (median follow-up 5.5 years).

3. Deaths from metastases. During 1960-88, 31 patients $(38 \cdot 3 \%)$ died, 15 of them (18.5\%) from proved metastases (10 female, 5 male patients, Fig 2). In 10 cases metastases were diagnosed clinically; in five cases necropsy was performed. One patient died from hepatic carcinoma as a second malignant tumour. In 10 cases it was noted that the regional lymphnodes were involved before the patients died from general haematogenous metastases.

As shown in Figure 3 most of our patients died within five years of their first treatment. One patient died 11 years and another even 25 years later from proved metastases of the primary conjunctival melanoma. Deaths from metastases occurred at an average of 6.2 years (median 5 years) after the first treatment of the lesion. The patients' average age was 67 (minimum 33, maximum 82 ) years.

The preponderance of 11 advanced tumours of more than $2.0 \mathrm{~mm}$ thickness in those patients who died from metastases was significant $\left(\chi^{2}=4 \cdot 07\right.$, $\mathrm{p}<0.05$ )

SURVIVAL RATE

The survival rate was calculated by the life table method. It compared the cumulative survival

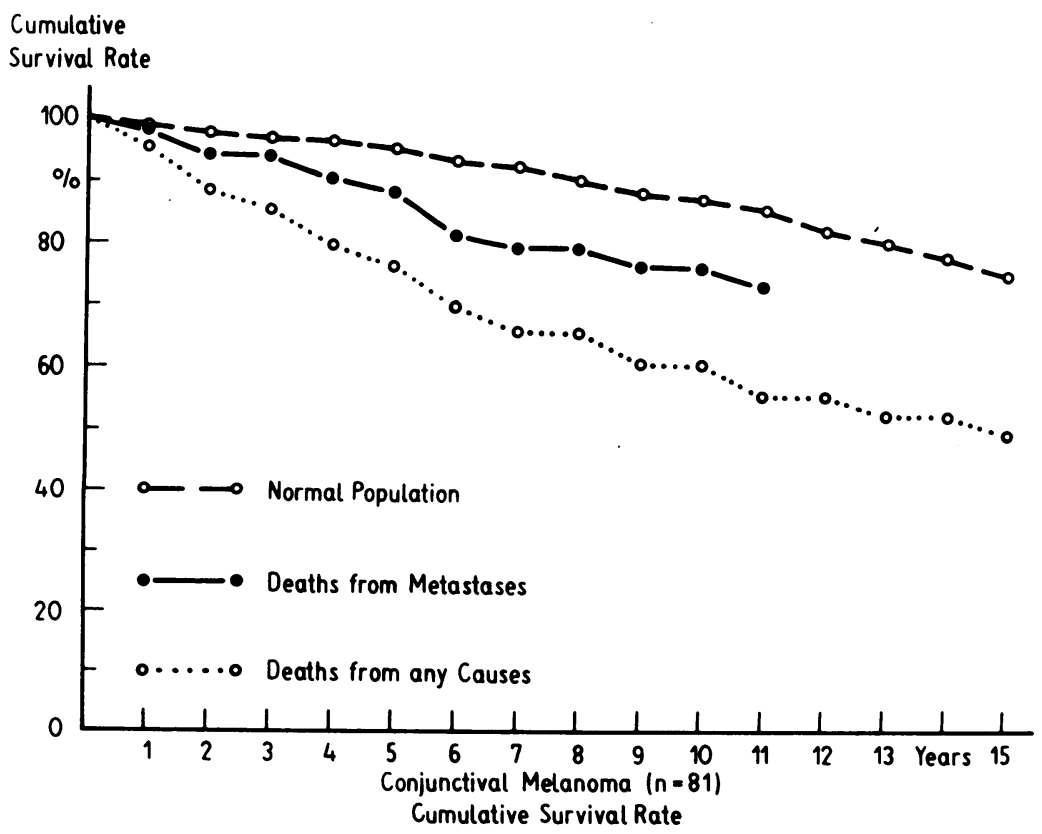

Figure 4 Cumulative survival rates of 81 patients with conjunctival melanoma in comparison with a population aged 55 years on the average.

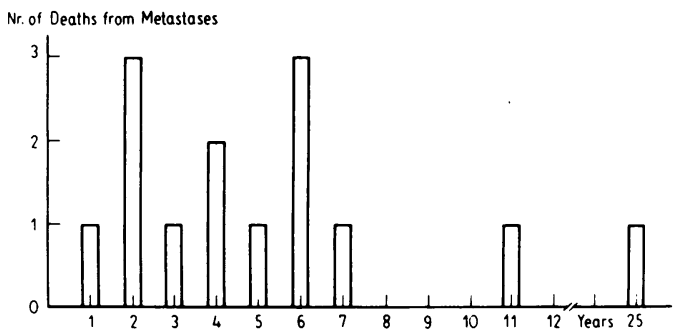

Figure 3 Metastatic deaths after beginning of treatment of 81 patients with conjunctival melanoma.

rates of our patients with those of a population aged 55 on the average. An overall cumulative survival rate of $76 \%$ after 5 years and $60 \%$ after 10 years was obtained. As regards deaths caused by metastases only, the cumulative survival rates were $87 \cdot 6 \%$ after 5 years and $76 \cdot 3 \%$ after 10 years (Fig 4).

\section{TUMOUR EXTENT AND PROGNOSIS}

Jakobiec $^{10}$ commented that tumour thickness is the 'sole sovereign prognosticator in conjunctival melanomas.' We therefore compared 36 small tumours (less than one quadrant of the bulbar conjunctiva and less than $2 \mathrm{~mm}$ thick) with 35 larger ones (more than one quadrant of the bulbar conjunctiva and/or more than $2 \mathrm{~mm}$ thick). This statistic includes only 71 cases with well defined postsurgical histopathological classification. As shown in Fig 5, the metastasis rate of the group with large tumours was $31 \cdot 4 \%$, almost three times higher than $11 \cdot 1 \%$ of the group with small melanomas. This higher rate of larger tumours is statistically significant $\left(\chi^{2}=4 \cdot 40\right.$; $\mathrm{p}<0.05)$.

Figure 6 shows the result of a comparison between the cumulative survival rates of 38 patients with small tumours and those of 43 patients with an advanced lesion. After a followup period of eight years there was a statistically (Mantel-Haenszel test, $\chi^{2}=4 \cdot 15 ; \mathrm{p}<0.05$ ) higher survival of the group with smaller tumours $(88.5 \%$, SE $8.7 \%)$ than of that with advanced melanomas $(65 \cdot 0 \%$, SE $10 \cdot 6 \%)$.

\section{TUMOUR ORIGIN AND PROGNOSIS}

The metastasis rates according to the origin of the melanoma were as follows:

Melanoma derived from naevus ................ 14.3\% (3 patients) Melanoma derived from PAM .................. 33.3\% (7 patients) Melanoma derived 'de novo' .................. 20.0\% (5 patients)

The apparently higher incidence of melanomas derived from PAM in the patients with metastases is not statistically significant $\left(\chi^{2}=2 \cdot 32 ; p>0.05\right.$; $\mathrm{df}=2$ ).

\section{THERAPEUTIC SIDE EFFECTS}

Local irradiation with $\beta$ applicators (Figs 7A-E) and cryotherapy with liquid nitrogen have lethal effects not only on tumour cells but also on normal surrounding tissue. To keep side effects within reasonable limits excessive treatment should be avoided. The following side effects could be observed: Phthisis bulbi 1 (after cryotherapy); cataract 2; local lens opacity 7 (after $\beta$ irradiation); symblepharon 2 (after cryotherapy); 


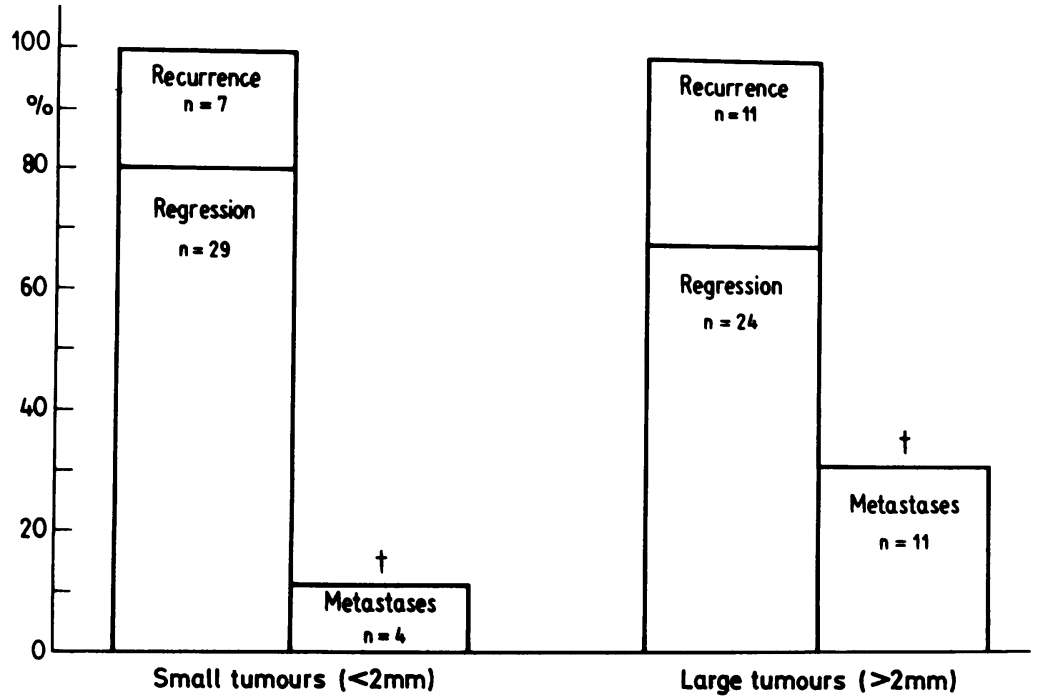

Figure 5 Regression, recurrence, and metastases after treatment of 36 small conjunctival melanomas in comparison with 35 larger ones.

corneal ulceration, descemetocele 2 (after cryotherapy); extented teleangiectasia of conjunctival vessels 3 ; iris atrophy 1 (after cryotherapy).

\section{Discussion}

It seems generally accepted that the development of malignant melanoma of the conjunctiva comprises three major groups. The lesions may derive from primary acquired melanosis, or from a pre-existing naevus, or develop 'de novo' where no antecedent lesion has been recognised. Therefore the pathologist needs a reliable clinical history for a comprehensive histological diagnosis, including the possible development of the tumour. This view may very well be an oversimplification, because Folberg et al.${ }^{11}$ found with the aid of further incisions of the lesions an associated naevus in approximately one-third of all melanomas developed from acquired melanosis. Some authors have related the prognosis of melanomas to their origin. Thus Yanoff and Fine $^{12}$ state that the mortality resulting from melanomas arising from naevi is $20 \%$, whereas

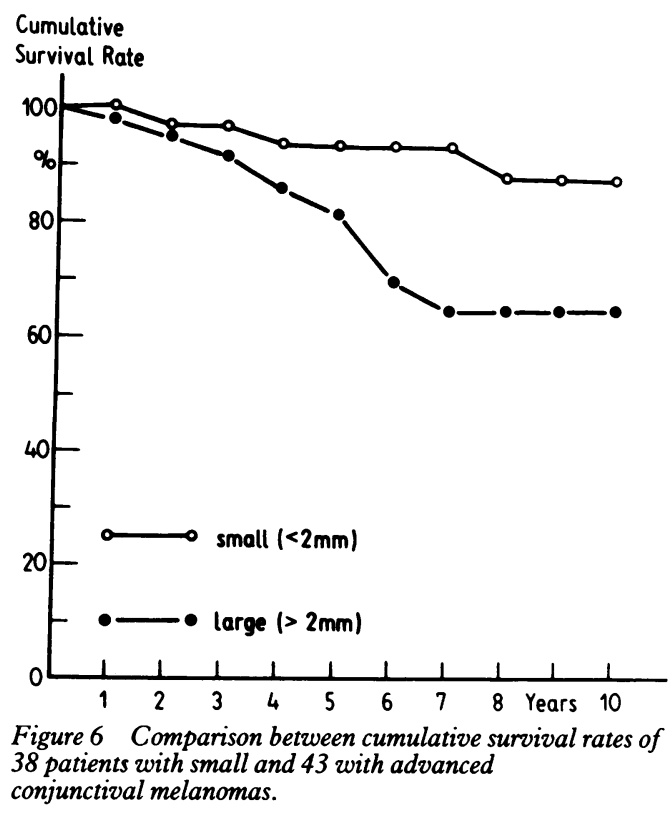

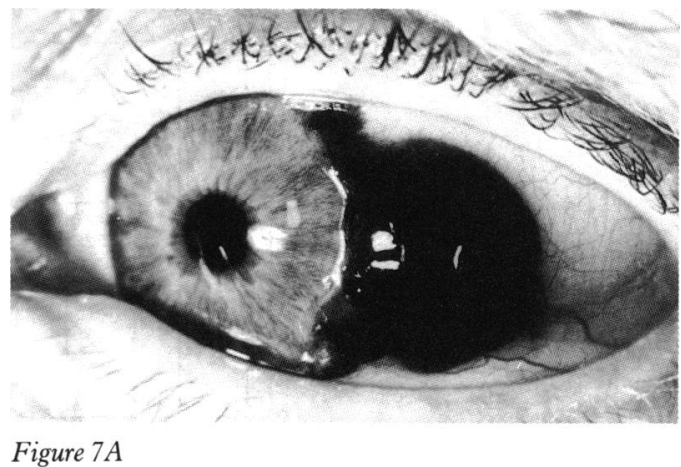

Figure 7 (A) Conjunctival melanoma of an only eye, female patient aged 62 years. $(B)$ Six months after brachytherapy patient aged 62 years. (B) Six months after brachytherapy
with $S r-90 / Y-90,150 G y .(C)$ Two years after treatment. $(D)$ Eight years after treatment. $(E) 11$ years after treatment, visual acuity $1 \cdot 0$. No recurrence or metastases 15 years after irradiation.

from melanomas arising from acquired melanosis or de novo it is $40 \%$. Conjunctival melanomas have both spindle and epithelioid cell types. The latter do not have such an important influence on the degree of malignancy as in choroidal melanomas.

The presence of a malignant melanocytic naevus is associated with a more favourable prognosis in patients with cutaneous melanomas. In contrast to this, Folberg $e t a l^{11}$ detected no such association between a better prognosis and the presence of a naevus in their series of conjunctival melanomas. In our cases the metastasis rates of melanomas developed from pre-existing naevi were $14 \cdot 3 \%$, de novo $20 \cdot 0 \%$, and from a primary acquired melanoma $33 \cdot 3 \%$. However, the highest metastasis rate of melanomas developing from PAM was statistically insignificant. We agree with Jakobiec, ${ }^{10}$ Zimmerman, ${ }^{13}$ and Folberg et al ${ }^{11}$ not to apply the classification of cutaneous melanomas to the study of conjunctival melanomas. In particular the absence of stratification in the conjunctival substantia propria precludes the use of the microstaging system of Clark et $\mathrm{al}^{14}$ in conjunctival melanomas.

Jakobiec ${ }^{10}$ commented that tumour thickness is the most important prognosticator in conjunctival melanomas. Our results confirm this statement. The metastasis rate of conjunctival melanomas larger than $2 \mathrm{~mm}$ thick or involvement of fornix, palpebral conjunctiva, caruncle, or cornea was $31.4 \%$ and significantly higher than that of the group with smaller tumours, with $11 \cdot 1 \%$. Folberg et $a l^{11}$ conclude from their careful evaluation of 131 patients with conjunctival melanoma that all lesions measuring at their

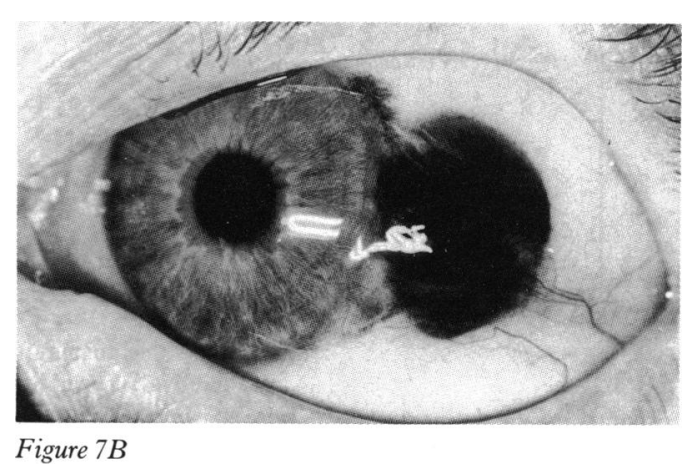




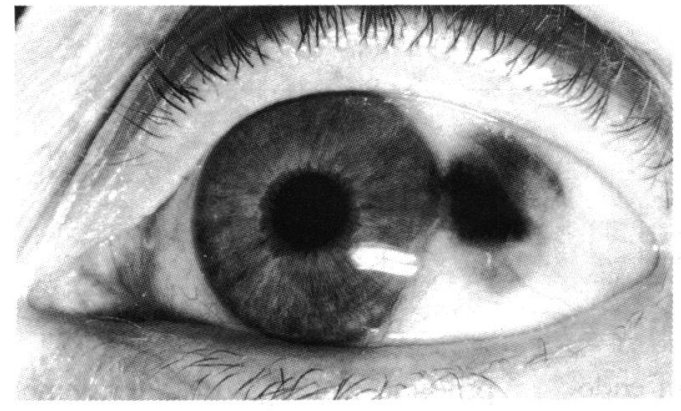

Figure $7 C$

greatest extent more than $0.8 \mathrm{~mm}$ are at high risk for the development of metastases - $29 \%$ and more. Though our findings are based on different material and methods, they are comparable with these results.

We can conclude that all lesions involving more than one quadrant of the bulbar conjunctiva and more than $2 \mathrm{~mm}$ thick are at high risk for development of metastases. On the other hand we observed lethal metastases in very small conjunctival melanomas and no metastases even after very thick tumours which had to be removed by exenteration. Thus it remains impossible for the ophthalmologist to obtain reliable prognostic signs to advise an individual patient. Both the evolution of primary acquired melanosis, which may wax and wane, ${ }^{15}$ and the behaviour of conjunctival melanomas will often remain unpredictable.

The cumulative survival rate was $87 \cdot 6 \%$ after 5 years and $76 \cdot 3 \%$ after 10 years when only deaths caused by metastases are considered. These results come very close to the survival rates found by Folberg et al. ${ }^{11}$ The overall mortality rate from metastases only in the 81 cases presented was $18.5 \%$, and, from all causes of death, $38.3 \%$. This is similar to the mortality rate of $26 \%$ published by Folberg et al. ${ }^{11}$ This statistical analysis of the actuarial curves for two groups of our patients indicates that there is a significant difference between small and larger tumours during a follow-up of eight years. With $88 \cdot 5 \%$ (SE $6.5 \%)$, small tumours give a better chance of survival than larger ones, with $65 \%$ (SE 10.6\%) $\left(\chi^{2}=4 \cdot 15 ; \mathrm{p}<0.005\right)$.

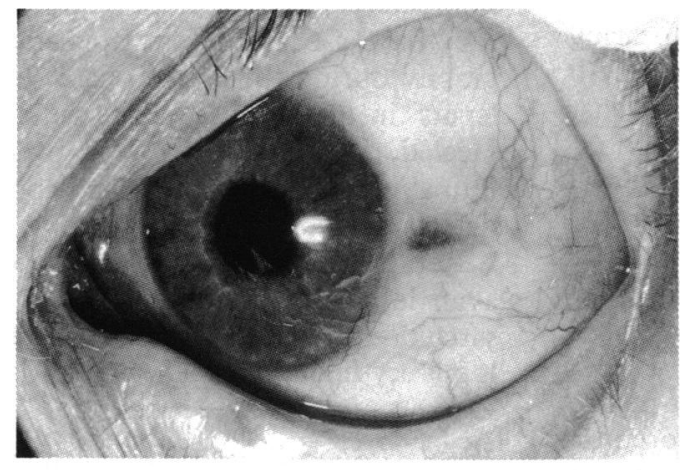

Figure $7 D$



Figure 7E

Many recent attempts have been made in this subject to find reliable prognostic indicators to predict whether a particular conjunctival melanoma is likely to recur or to metastasize. 11617 From the findings of the authors cited and the clinical observation presented in this paper it can be concluded that thickness and location of the neoplasm, its mitotic rate or the histopathological feature of an associated acquired melanosis must be regarded as prognostic signs. An international multicentric collaborative study would perhaps be suited to throw light on the unpredictable behaviour of this tumour.

1 Henkind D. Conjunctival melanocytic lesions: natural history. In: Jakobiec FA, ed. Ocular and adnexal tumors. Birmingham, Alabama: Aesculapius, 1978; 572-82.

2 Notter G. Das maligne epibulbäre Melanom. Strahlentherapie 1955; 96: 518-537.

3 Lederman $M$. Radiotherapy of malignant melanomata of the eye. Br F Radiol 1961; 34: 21-42.

4 Lommatzsch P. Beta irradiation of conjunctival melanomas. Trans Ophthalmol Soc UK 1977; 97: 378-80.

5 Lommatzsch P. Beta-ray treatment of malignant epibulbar melanoma. Graefes Arch Klin Exp Ophthalmol 1978; 209 111-24.

6 Jakobiec FA, Brownstein S, Wilkinson RD. Katzin HM Adjuvant cryotherapy for focal nodular melanoma of the conjunctiva. Arch Ophthalmol 1982; 100: 115-8.

7 Harmer MH, Oosterhuis JA. TNM classification of ophthalmic tumours. Geneva: International Union Against Cancer, 1985.

Kimours. Gira. In Behandlung Behandlung des malignen Melanoms der Aderhaut: Vergleich von konservativer Therapie (106-Ru/106-RhApplikator) und Enukleation ohne und mit postoperative Orbitabestrahlung, 1960-1979. Klin Monatsbl Augenheilkd
1984; 184: 2-14.

9 1984; 184: $2-14$.

Lommatzsch PK. $\beta$-irradiation of choroidal melanoma with 106-Ru/106-Rh-applicators. Arch Ophthalmol 1983; 101 713-7.

10 Jakobiec FA. Conjunctival melanoma: unfinished business Arch Ophthalmol 1981; 98: 1378-84.

11 Folberg R, McLean IW, Zimmerman LE. Malignant mela noma of the conjunctiva. Hum Pathol 1985; 16: 136-43

12 Yanoff M, Fine BS. Ocular pathology. A text and atlas. Philadelphia: Harper and Row, 1982: 800 .

13 Zimmerman LE. The histogenesis of conjunctival melanomas. In: Jakobiec FA, ed. Ocular and adnexal tumors. Birmingham, Alabama: Aesculapius, 1978: 572-82.

14 Clark WH Jr, Folberg R, Ainsworth AM. Tumor progression in primary human cutaneous malignant melanomas. In: malignant melanoma. New York: Grune and Stratton, 1979; malignant.

15 Spencer WH, Zimmerman LE. Conjunctiva. In: Spencer WH, ed. Ophthalmic pathology. An atlas and textbook. Philadelphia, London, Toronto; Saunders, 1985: 1: Philadelp

16 Fuchs U, Kivelä T, Liesto K, Tarkkanen A. Prognosis of conjunctival melanomas in relation to histopathological features. Br f Cancer 1989; 59: 261-7.

17 Stefani FH. A prognostic index for patients with malignant melanoma of the conjunctiva. Graefes Arch Clin Exp Ophthalmol 1986; 224: 580-2. 